\title{
CORRIENTE ALTERNA
}
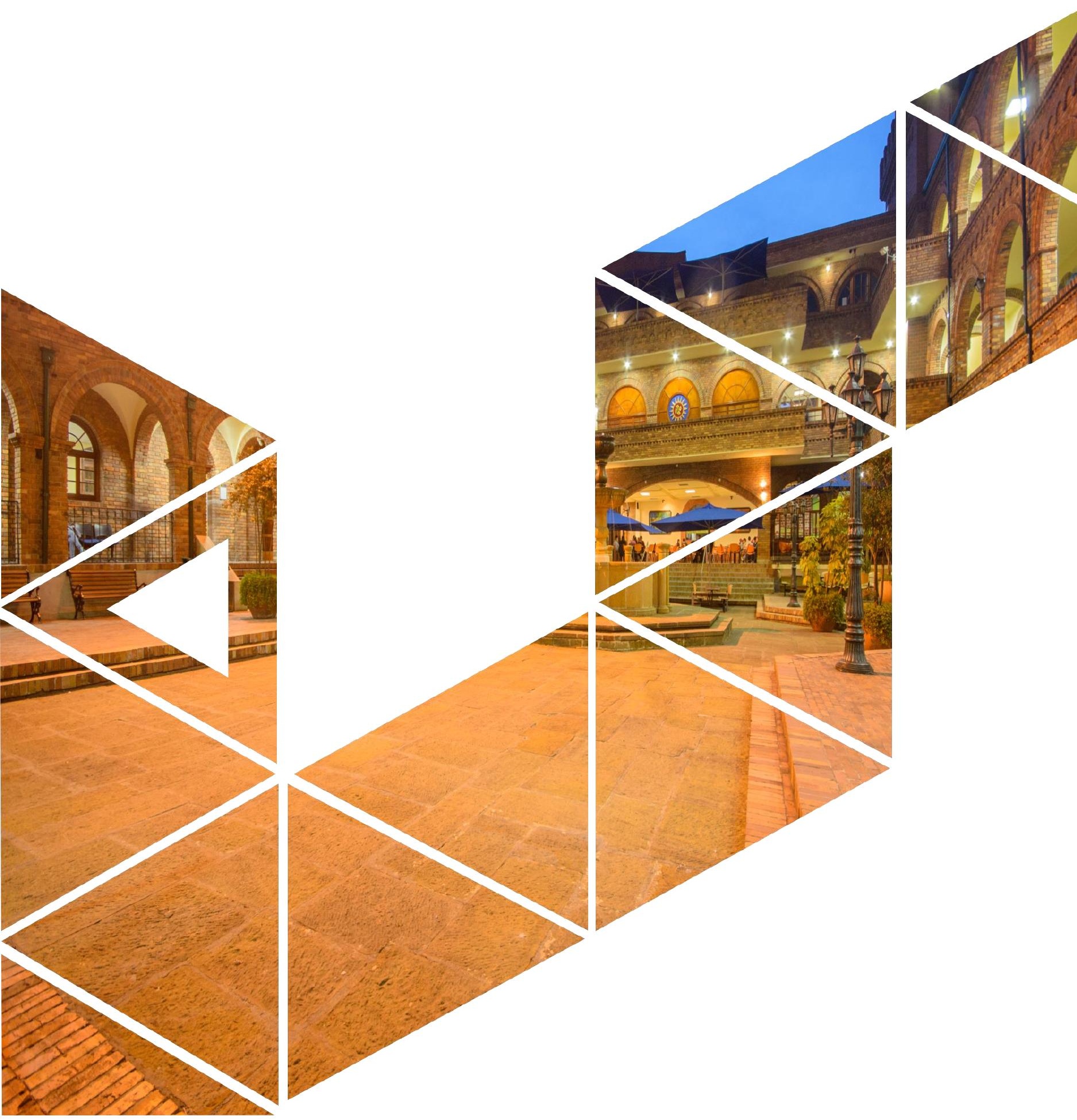


\section{FICHA TÉCNICA}

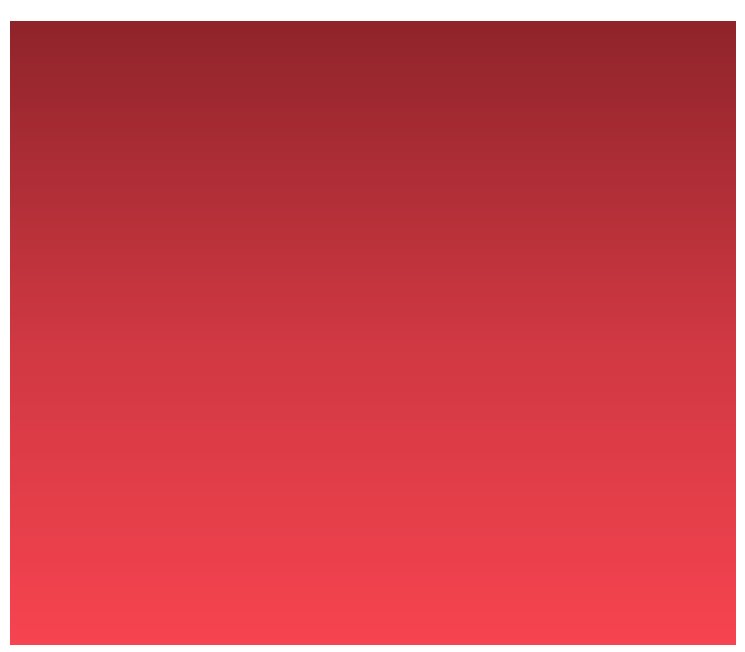

隥

Periodo de análisis: hasta 2019

Fecha de consulta: 26/02/2020

Herramientas: VantagePoint

(24) $\Omega$ versión 11, Microsoft Excel, Harzing's Publish or Peris

versión 7.12.257.7189

Datos abiertos:

DOI: 10.5281/zenodo.3926673
EXPRESIÓN DE BÚSQUEDA SCIELO CITATION INDEX - WEB OF SCIENCE

$(O O=$ universidad santo tomas AND $\mathrm{CU}=\mathrm{COLOMBIA}) \quad \mathrm{OR} \quad(\mathrm{OO}=$ saint thomas aquinas univer sity $A N D C U=C O L O M B I A)$ OR (OO=saint thomas university AND $\mathrm{CU}=\mathrm{COLOMBIA}) \quad \mathrm{OR} \quad(\mathrm{OO}=$ santo tomás university AND $C U=$ COLOMBIA) OR $(O O=$ santo tomas university AND $\mathrm{CU}=\mathrm{COLOMBIA}) \quad \mathrm{OR} \quad(\mathrm{OO}=$ Univ Santo Tomas AND CU=COLOMBIA) NOT ((OO=Univ Santo Tomas AND $\mathrm{CU}=$ Philippines) $\mathrm{OR}$ (OO=Univ Santo Tomas Hosp AND CU=Philippines) OR (OO=Univ Santo Tomas AND CU=Chile))

Refinado por: [excluyendo] AÑOS DE PUBLICACIÓN: ( 2020 )

Período de tiempo: Todos los años Índices: SCIELO

Más información:

DATOS USTA

https://observatoriocienciometria.usta.edu.co/index.php/metricas-usta/

corriente-alternativa 


\section{Corriente ALTERNA}

Olga Lucia Ostos Ortiz

iD ORCID 0000-0002-6477-9872

Nicolas Londoño Bernal

(iD) ORCID 0000-0002-5621-4521

La llamada Corriente Alterna en el ámbito de las publicaciones científicas, y específicamente referida a Latinoamérica, se refiere al grupo de publicaciones científicas, indexadores, y bibliotecas digitales que permiten la visibilidad e impacto de la ciencia en una apuesta de acceso abierto e impacto regional. En este sentido, pretender reducir la distancia que toma respecto a los indexadores internacionales de corriente principal como lo son las grandes compañías de Elsevier y Clarivate Analytics con sus plataformas Scopus y Web Of Science (Aparicio Gómez \& Ostos Ortíz, 2018).

Entre las plataformas y apuestas más reconocidas a nivel internacional en corriente alterna se encuentran SciELO y Redalyc. Estas plataformas constituyen librerías electrónicas e indexadores en Latinoamérica que aseguran acceso a revistas y producción académica con criterios de calidad, a la vez que enlazan la producción académica de libre acceso para la región. 
La Universidad Santo Tomás ha sumado esfuerzos en los últimos años de cara a la apuesta regional por la ciencia abierta (Open Access). En ese sentido, reconoce la importancia de invitar a sus autores a la publicación de la producción académica en revistas que cumplan esta condición y que además garanticen un impacto y crecimiento del conocimiento científico y artístico regional (Aparicio Gómez et al., 2019). La apuesta regional por un conocimiento producido desde Latinoamérica con atención a las propias realidades y a la vez con proyección mundial ha visto los frutos del esfuerzo de la región latinoamericana en el compromiso con la producción de ciencia de impacto y de acceso democrático. Por ello, se presentan a continuación los resultados de análisis de la producción académica en corriente alterna. Se analizan los resultados para la SciELO entre 2003 y 2019. Se ha excluido los resultados de Redalyc debido a la reciente actualización de la herramienta Harzing's Publish or Peris en la que no fue posible usar el operador Site de Google en la recolección de datos.

Las publicaciones académicas muestran un aumento en número y citación en revistas en el índice en los últimos años. Para 2019 se cuenta con cerca de 60 publicaciones en revistas en el índice, y un aproximado de 80 citas que se consolidan en el índice de medición de impacto H sobre 8,5. La Universidad Santo Tomás continúa aportando de manera activa al fortalecimiento de la producción académica nacional de alto impacto para atender y mejorar la calidad de vida de la región.

La producción de la Universidad Santo Tomás indexada en SciELO se escribe mayormente en español, y entre las palabras de mayor concurrencia en las publicaciones encontramos "Colombia", "Educación", "niñez", y términos referenciados en el campo de la salud y la actividad física. Ello demuestra el enfoque nacional con perspectiva regional de las publicaciones en estas revistas, así como la atención a problemáticas de primera necesidad en la región latinoamericana (Ostos-Ortíz, 2019).

Las áreas con mayor volumen de publicación son psicología e ingenierías, seguido del área de ciencias sociales y las artes - humanidades. La tendencia continúa con respecto a reportes de producción de años anteriores y corresponde al énfasis de la USTA como comunidad académica. Se resalta nuevamente la producción en las áreas de ingeniería y su apuesta por la ciencia abierta en la universidad (Corchuelo-Rodriguez et al., 2019).

En cuanto a revistas con más publicaciones de autores USTA ancladas en el índice, lidera la revista "Diversitas: perspectivas en Psicología" con 69 publicaciones. Un logro consecuente con el énfasis iberoamericano de la publicación periódica, y las redes regionales que ha logrado tejer. Le siguen en orden de publicaciones "Nutrición Hospitalaria" y el "International Journal of Morphology". 


\section{METODOLOGÍA}

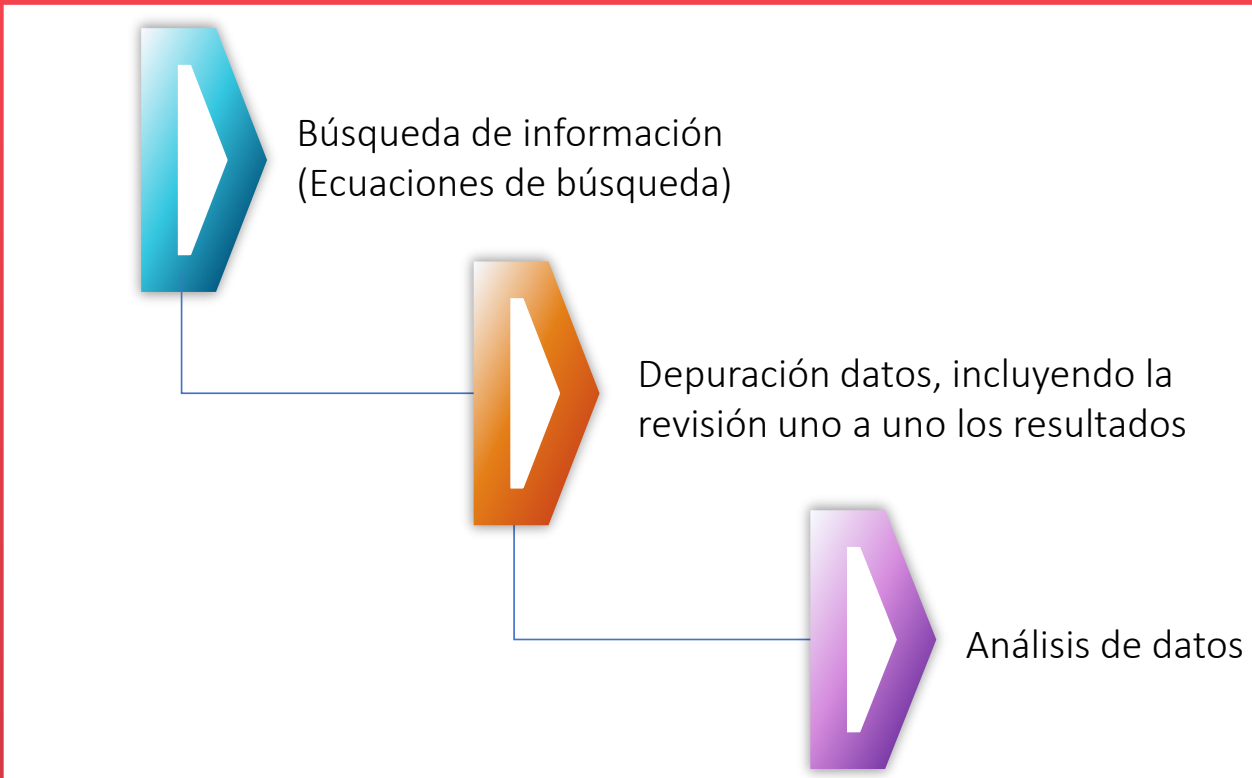

Con respecto a la primera publicación de este boletín se hicieron dos ajustes para garantizar a integridad y calidad de los datos, así:

- No se integraron resultados de Redalyc dado que con la actualización de la Harzing's Publish or Peris no se pudo usar el operado Site de Google para garantizar la recolección de los datos.

- Se usó el Índice SciELO de WoS para la consulta unificada de bibliografía.

Nota: invitamos a la comunidad académica a conocer y consultar los indicadores propuestos por Redalyc en el camino hacia consolidación del acceso abierto de la región y el uso métricas responsables, ingresando en: https://www.redalyc.org/institucion.oa?id=16295\&tipo=coleccion 


\section{RESULTADOS}

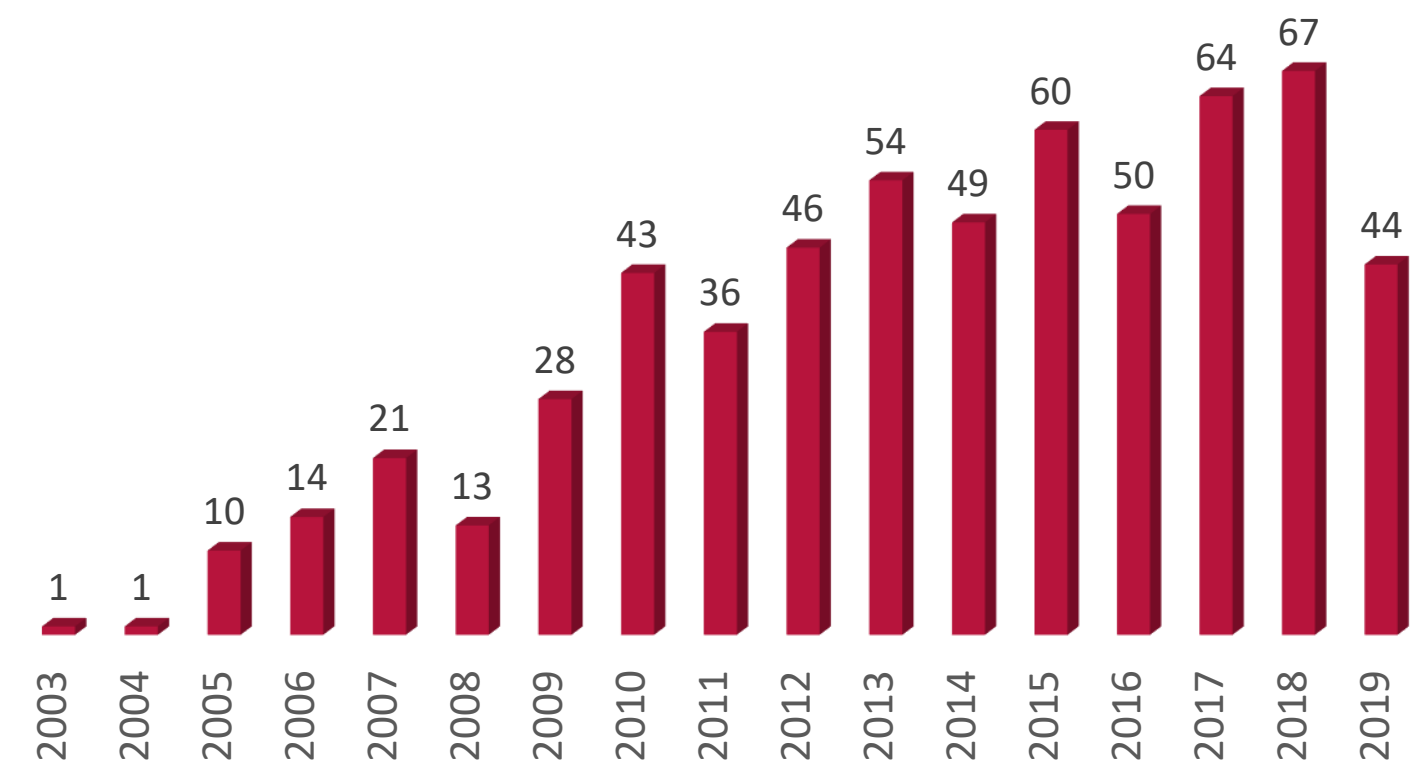

Gráfico 12. Publicaciones USTA en SciELO por año (2003-2019)

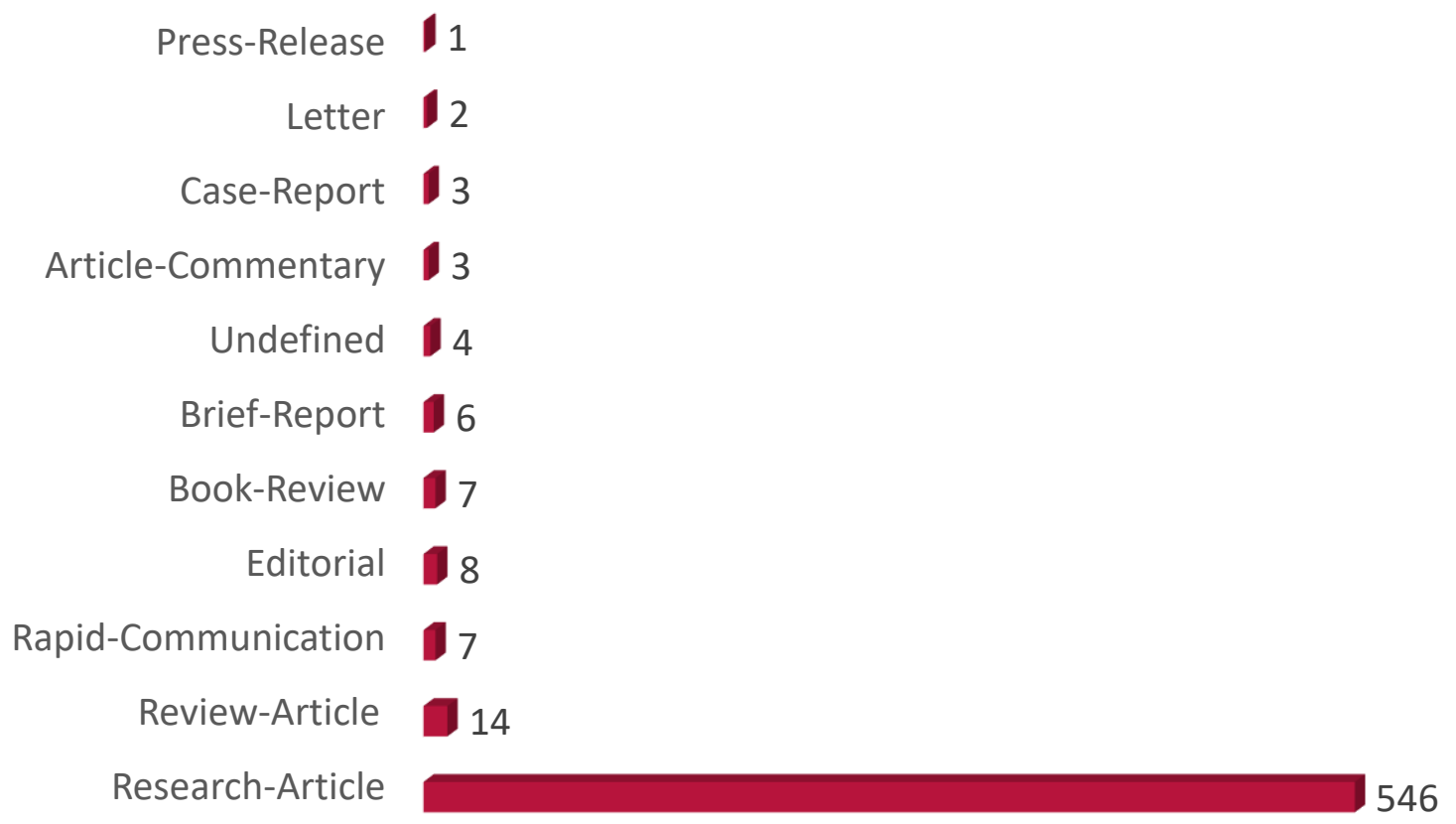

Gráfico 13. Publicaciones USTA por tipología en SciELO (2003-2019) 
531

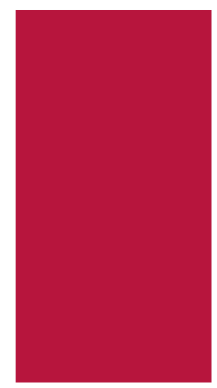

Español
68

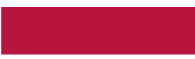

Inglés
2

Portugués

Gráfico 14. Publicaciones USTA por idioma en SciELO (2003-2019)

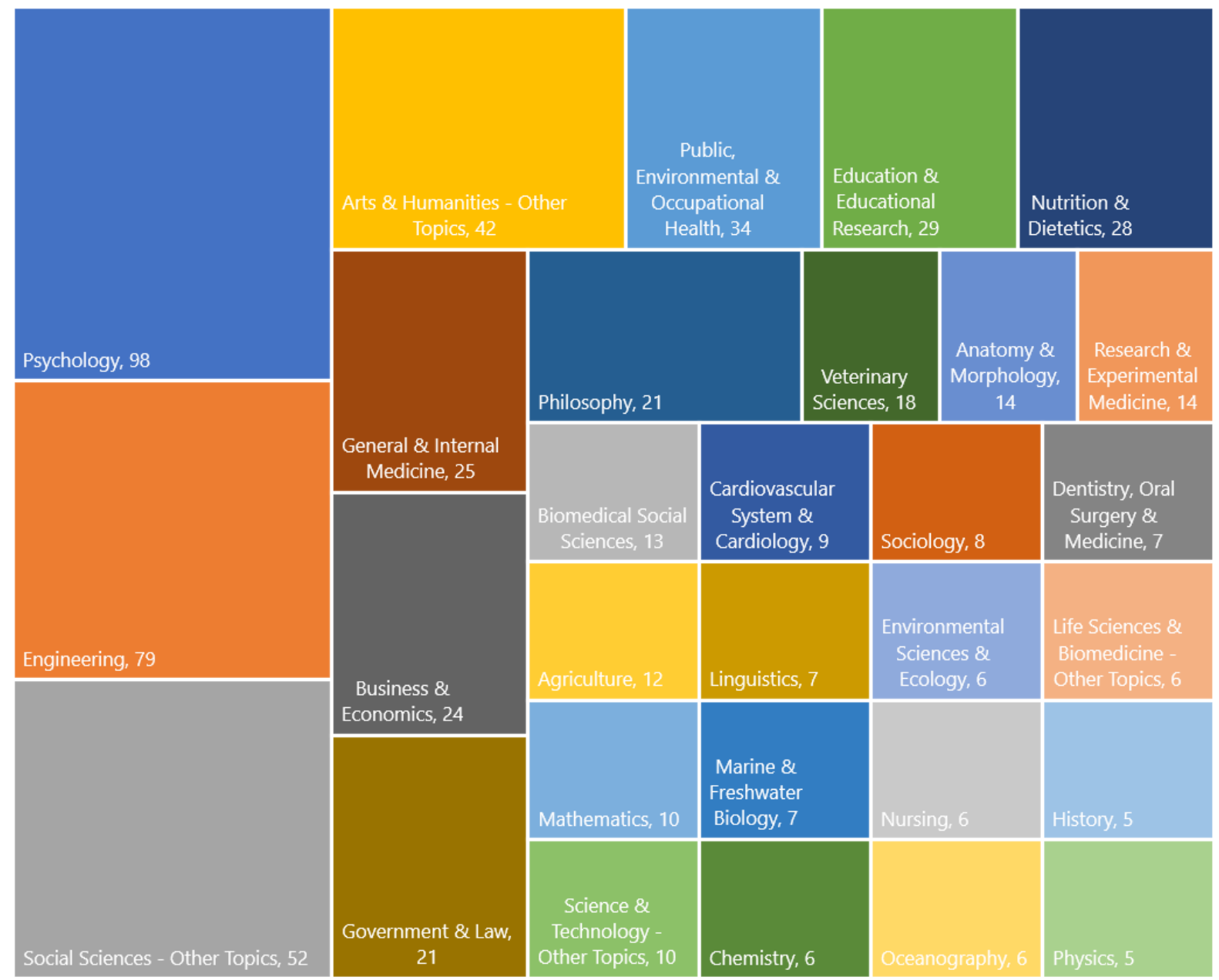

Gráfico 15. Publicaciones USTA en SciELO por áreas de conocimiento

Pág. 22 


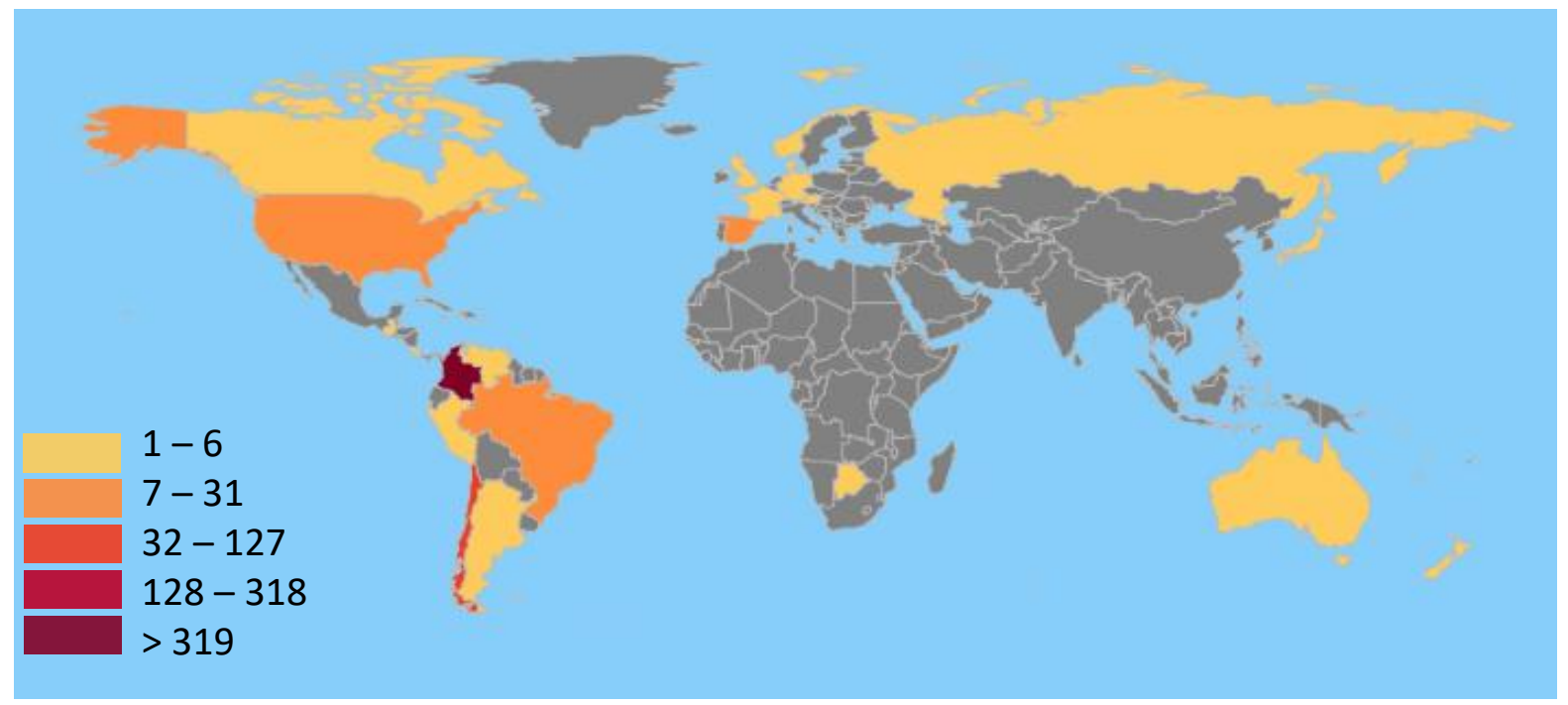

Gráfico 16. Mapa de Publicaciones USTA en SciELO por filiación de autor (2003-2019) 
120

- Publicaciones

- Citas

80

40

0

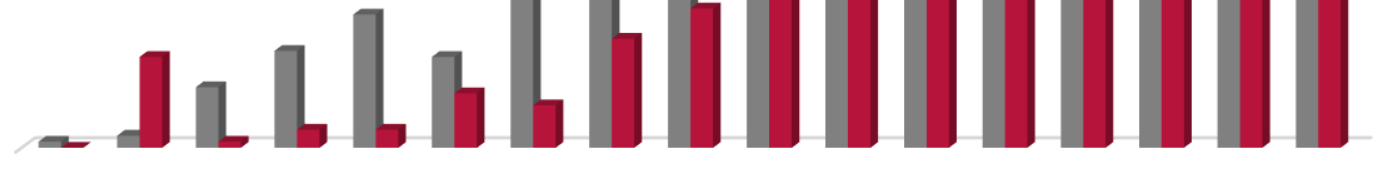

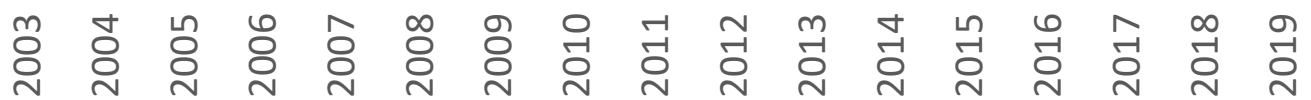

Gráfico 18. Citas y publicaciones de las publicaciones USTA en SciELO (2003-2019)

TMBLA Indicadores bibliométricos de las publicaciones 5 USTA en SciELO (2003-2019)

\begin{tabular}{|c|c|c|c|c|r|}
\hline \multicolumn{4}{|c|}{ Citas } & \multicolumn{3}{c|}{ Índice } \\
\hline Totales & Por año & Por documento & H & G & HC \\
\hline 505 & 28,7 & 1,4 & 8,5 & 10,5 & 5 \\
\hline
\end{tabular}




\begin{tabular}{|c|c|c|c|c|}
\hline Citas & Autor & Título & Año & Recurso \\
\hline 21 & $\begin{array}{l}\text { Françoise Contreras, Juan Carlos } \\
\text { Espinosa, Gustavo Esguerra, } \\
\text { Andrea Haikal, Alejandra Polanía, } \\
\text { Adriana Rodríguez }\end{array}$ & $\begin{array}{l}\text { Autoeficacia, ansiedad y } \\
\text { rendimiento académico en } \\
\text { adolescentes }\end{array}$ & 2005 & $\begin{array}{l}\text { Diversitas: } \\
\text { Perspectivas en } \\
\text { Psicología }\end{array}$ \\
\hline 18 & $\begin{array}{l}\text { Françoise Contreras, Gustavo A. } \\
\text { Esguerra, Juan Carlos Espinosa, } \\
\text { Viviana Gómez }\end{array}$ & $\begin{array}{l}\text { Estilos de afrontamiento y calidad } \\
\text { de vida en pacientes con } \\
\text { insuficiencia renal crónica (irc) en } \\
\text { tratamiento de hemodiálisis }\end{array}$ & 2007 & $\begin{array}{l}\text { Acta Colombiana } \\
\text { de Psicología }\end{array}$ \\
\hline 14 & $\begin{array}{l}\text { Wilson López López, Luis Manuel } \\
\text { Silva, María Caridad García- } \\
\text { Cepero, María Constanza Aguilar } \\
\text { Bustamante, Eduardo Aguado } \\
\text { López }\end{array}$ & $\begin{array}{l}\text { Retos para la colaboración nacional } \\
\text { e internacional en la psicología } \\
\text { latinoamericana: un análisis del } \\
\text { sistema Redalyc, } 2005-2007\end{array}$ & 2011 & $\begin{array}{l}\text { Estudos de } \\
\text { Psicologia (Natal) }\end{array}$ \\
\hline 13 & $\begin{array}{l}\text { Daniel Humberto Prieto- } \\
\text { Benavides, Jorge Enrique Correa- } \\
\text { Bautista, Robinson Ramírez-Vélez }\end{array}$ & $\begin{array}{l}\text { Niveles de actividad física, condición } \\
\text { física y tiempo en pantallas en } \\
\text { escolares de Bogotá, Colombia: } \\
\text { estudio FUPRECOL }\end{array}$ & 2015 & $\begin{array}{l}\text { Nutrición } \\
\text { Hospitalaria }\end{array}$ \\
\hline 13 & $\begin{array}{l}\text { Gustavo Esguerra Pérez, Pablo } \\
\text { Guerrero Ospina }\end{array}$ & $\begin{array}{l}\text { Estilos de aprendizaje y rendimiento } \\
\text { académico en estudiantes de } \\
\text { Psicología }\end{array}$ & 2010 & $\begin{array}{l}\text { Diversitas: } \\
\text { Perspectivas en } \\
\text { Psicología }\end{array}$ \\
\hline 12 & $\begin{array}{l}\text { Brenda Isabel López-Vargas, } \\
\text { Sandra Patricia Basto-Torrado }\end{array}$ & $\begin{array}{l}\text { Desde las teorías implícitas a la } \\
\text { docencia como práctica reflexiva }\end{array}$ & 2010 & $\begin{array}{l}\text { Educación y } \\
\text { Educadores }\end{array}$ \\
\hline 12 & $\begin{array}{l}\text { Francoise Contreras, Gustavo } \\
\text { Esguerra }\end{array}$ & $\begin{array}{l}\text { Psicología positiva: una nueva } \\
\text { perspectiva en psicología }\end{array}$ & 2006 & $\begin{array}{l}\text { Diversitas: } \\
\text { Perspectivas en } \\
\text { Psicología }\end{array}$ \\
\hline 10 & $\begin{array}{l}\text { Robinson Ramírez-Vélez, } \\
\text { Katherine González-Ruíz, Jorge } \\
\text { Enrique Correa-Bautista, Javier } \\
\text { Martínez-Torres, José F. } \\
\text { Meneses-Echavez, David Rincón- } \\
\text { Pabón }\end{array}$ & $\begin{array}{l}\text { Ferritin levels in pregnant } \\
\text { Colombian women }\end{array}$ & 2015 & $\begin{array}{l}\text { Nutrición } \\
\text { Hospitalaria }\end{array}$ \\
\hline 10 & $\begin{array}{l}\text { Robinson Ramírez-Vélez, José F. } \\
\text { Meneses-Echavez, Katherine } \\
\text { González-Ruíz, Jorge Enrique } \\
\text { Correa }\end{array}$ & $\begin{array}{l}\text { Fitness muscular y riesgo cardio- } \\
\text { metabólico en adultos jóvenes } \\
\text { colombianos }\end{array}$ & 2014 & $\begin{array}{l}\text { Nutrición } \\
\text { Hospitalaria }\end{array}$ \\
\hline 9 & $\begin{array}{l}\text { Juan Carlos Aguirre García, Luis } \\
\text { Guillermo Jaramillo Echeverri }\end{array}$ & $\begin{array}{l}\text { EL OTRO EN LÉVINAS: Una salida a la } \\
\text { encrucijada sujeto-objeto y su } \\
\text { pertinencia en las ciencias sociales }\end{array}$ & 2006 & $\begin{array}{l}\text { Revista } \\
\text { Latinoamericana de } \\
\text { Ciencias Sociales, } \\
\text { Niñez y Juventud }\end{array}$ \\
\hline
\end{tabular}


TABLA Top 10 de las revistas con más publicaciones

1 USTA en SCIELO (2003-2019)

\begin{tabular}{|c|l|}
\hline $\begin{array}{c}\text { N.o de publicaciones } \\
69\end{array}$ & \multicolumn{1}{c|}{ Revista } \\
\hline 21 & Diversitas: Perspectivas en Psicología \\
\hline 14 & Nutrición Hospitalaria \\
\hline 14 & International Journal of Morphology \\
\hline 12 & Iteckne \\
\hline 12 & Hallazgos \\
\hline 11 & Archivos de medicina veterinaria \\
\hline 11 & Revista Cubana de Investigaciones Biomédicas \\
\hline 9 & DYNA \\
\hline 9 & Revista Colombiana de Estadística \\
\hline 9 & Cuadernos de Contabilidad \\
\hline & Revista de Salud Pública \\
\hline
\end{tabular}

TABLA Top autores USTA con publicaciones en SciELO $8 \quad(1987-2019)$

\begin{tabular}{|l|c|}
\multicolumn{1}{c|}{ Autor } & Publicaciones \\
\hline Meneses-Echavez, J. F. & 5 \\
\hline Patiño-Jacinto, Ruth Alejandra & 4 \\
\hline Jaramillo Pérez, Jorge Mario & 4 \\
\hline López-Vaca, Oscar Rodrigo & 4 \\
\hline Santamaría-Rodríguez, Juan Esteban & \\
\hline
\end{tabular}




\section{Referencias bibliográficas}

Abelson, P. (1990). Mechanisms for evaluating scientific information and the role of peer review. Journal of the American Society for Information Science, 41(3), 216-222. https://doi.org/10.1002/(SICI)1097-4571(199004)41:3<216::AIDASI13>3.0.CO;2-6

Aparicio Gómez, O. Y., \& Ostos Ortíz, O. L. (2018). Las TIC como herramientas cognitivas para la investigación. Revista Interamericana de Investigación, Educación y Pedagogía, RIIEP, 11(1), 81-86. https://doi.org/10.15332/s1657107X.2018.0001.08

Aparicio Gómez, O. Y., Ostos Ortíz, O. L., Gallego Cortes, M., \& Abadía García, C. (2019). Análisis sistemático de los artículos publicados en la revista interamericana de educación, investigación y pedagogía (RIIEP) durante 2014 a 2019. Revista Interamericana de Investigación, Educación y Pedagogía, RIIEP, 12(2), 225-240. https://doi.org/10.15332/25005421.5015

Batista, P. D., Campiteli, M. G., \& Kinouchi, O. (2006). Is it possible to compare researchers with different scientific interests? Scientometrics, 68(1), 179-189. https://doi.org/10.1007/s11192-006-0090-4

Bunge, M. (2018). La ciencia: Su método y su filosofía. Laetoli.

Carreño-Dueñas, D., Corchuelo-Rodriguez, C. A., \& Patacón-Ruiz, I. P. (2019a).

Revistas de ciencias sociales y el derecho en el modelo de clasificación de Publindex: Impacto de la revista científica. Verba luris, 42, 113-121.

https://doi.org/10.18041/0121-3474/verbaiuris.42.5662

CASRAI project. (2019). Snowball Metrics-STANDARDIZED RESEARCH METRICS - BY THE SECTOR FOR THE SECTOR. Snowball Metrics.

https://snowballmetrics.com/

Castelló-Cogollos, L., Sixto-Costoya, A., Lucas-Domínguez, R., Agulló-Calatayud, V., González De Dios, J., \& Aleixandre-Benavent, R. (2018). Bibliometrics and indicators of scientific activity (XI): Other useful resources in the evaluation: Google scholar, microsoft academic, Ifindr, dimensions and Lens.org. Acta Pediatrica Espanola, 76(9-10), 123-130. Scopus. 
CLACSO. (2019). ¿Qué es el FOLEC? https://www.clacso.org/folec/que-es-el-folec/

Coordinación del OCTS, O. E. I., Albornoz, M., de la RICYT, C., \& Barrere, R. (2017). Aspectos Conceptuales para la Medición de la Vinculación de la Universidad con el Entorno Socioeconómico (Manual de Valencia).

http://www.ricyt.org/wpcontent/uploads/2017/06/files manual vinculacion.pdf

Corchuelo-Rodriguez, C. A. (2018). Repositorio institucional como estrategia de visibilidad y gestión del conocimiento en el Modelo Nacional de CTel (Ciencia, Tecnología e Innovación).

Corchuelo-Rodriguez, C. A., Barreto-Montenegro, A. E., López-Báez, J. D., Ostos-Ortiz, O. L., Paez, L. M., Florian-Escobar, M. P., Castillo Medellín, D., Millán Ramírez, M. A., Garnica Posada, L. C., \& Prada Jiménez, C. A. (2019). Boletín bibliométrico USTA - No. 1 (2019). N. 1, 72. https://doi.org/10.15332/dt.inv.2019.00138

Corchuelo-Rodríguez, C. A., Patacón-Ruiz, I., \& Piza-Amado, K. (2020). Revistas de Ciencias Sociales en el modelo de clasificación de Publindex: Prospectiva de la convocatoria n. ${ }^{\circ} 830$ del 2018 en la fase III. Impacto de la revista científica. Revista Interamericana de Investigación, Educación y Pedagogía, RIIEP, 13(1), 137-155. https://doi.org/10.15332/25005421/5464

(Declaration on Research Assessment), D., \& Pardal-Peláez, B. (2018). Declaración de San Francisco sobre la evaluación de la investigación. Revista ORL, 9(4), 295. https://doi.org/10.14201/orl.17845

Egghe, L. (2006). Theory and practise of the g-index. Scientometrics, 69(1), 131-152. https://doi.org/10.1007/s11192-006-0144-7

Héder, M. (2017). From NASA to EU: the evolution of theTRL scale in Public Sector Innovation. https://www.innovation.cc/discussionpapers/2017 2223 heder nasa-to-eu-trl-scale.pdf

Hicks, D., Wouters, P., Waltman, L., de Rijcke, S., \& Rafols, I. (2015). Bibliometrics: The Leiden Manifesto for research metrics. Nature News, 520(7548), 429. https://doi.org/10.1038/520429a

Hirsch, J. E. (2005). An index to quantify an individual's scientific research output. Proceedings of the National Academy of Sciences of the United States of America, 102(46), 16569-16572. https://doi.org/10.1073/pnas.0507655102 
Katz, J. S., \& Martin, B. R. (1997). What is research collaboration? Research Policy, 26(1), 1-18. https://doi.org/10.1016/S0048-7333(96)00917-1

Kuhn, S. T. (2011). La estructura de las revoluciones científicas. Fondo de Cultura Económica.

López-Aguado, E., \& Arbeláez-Vargas, E. J. (2016). Reapropiación del conocimiento y descolonización: El acceso abierto como proceso de acción política del sur. Revista colombiana de sociología, 39(2), 69-88.

Lucas-Domínguez, R., Sixto-Costoya, A., Castelló Cogollos, L., González De Dios, J., \& Aleixandre-Benavent, R. (2018). Bibliometrics and indicators of scientific activity (IX). Scientometric indicators in Scopus. Analysis of publications on pediatrics. "Analyze search results» and "citation overview» function. Acta Pediatrica Espanola, 76(5-6), 90-96. Scopus.

Martin, B. R., \& Irvine, J. (1983). Assessing basic research: Some partial indicators of scientific progress in radio astronomy. Research Policy, 12(2), 61-90. https://doi.org/10.1016/0048-7333(83)90005-7

Matovelle, R. G., Galarraga, S. E., Lecuona, M. R., \& Hernandis, B. (2019). A bibliometric analysis of the relationship between design and innovation. Espacios, 40(22). Scopus.

OAST (Office of Aeronautical and Space Technology). (1991). Integrated Technology Plan for the Civil Space Program.

https://www.lpi.usra.edu/lunar/strategies/NASALunarArchitecture/exp tech plan.pdf

Organización Mundial de la Propiedad Industrial. (1967). https://www.wipo.int/about-wipo/es/index.html

Ostos-Ortíz, O. L. (2019). Revisión sistemática y análisis del enfoque y alcance de la revista interamericana de investigación y pedagogía.

Repiso, R. (2018). «Tabla periódica» de indicadores cienciométricos - EC3Metrics. https://ec3metrics.com/tabla-periodica-de-indicadores-cienciometricos/

Robinson-García, N., Repiso, R., \& Torres-Salinas, D. (2018). Perspectiva y retos de los profesionales de la evaluación científica y la bibliometría. El profesional de la información (EPI), 27(3), 461-466. https://doi.org/10.3145/epi.2018.may.01 
Rodríguez-García, A. M., Trujillo Torres, J. M., \& Sánchez Rodríguez, J. (2019). Impact of scientific productivity on digital competence of future teachers:

Bibliometric approach on Scopus and web of science. Revista Complutense de Educacion, 30(2), 623-646. Scopus. https://doi.org/10.5209/RCED.58862

Schreiber, M. (2008). EDITORIAL: To share the fame in a fair way, $h m$ modifies $h$ for multi-authored manuscripts. https://doi.org/10.1088/13672630/10/4/040201

Sidiropoulos, A., Katsaros, D., \& Manolopoulos, Y. (2006). Generalized h-index for Disclosing Latent Facts in Citation Networks. arXiv:cs/0607066. http://arxiv.org/abs/cs/0607066

Studies (CWTS), C. for S. and T. (2019). CWTS Leiden Ranking. CWTS Leiden Ranking; Centre for Science and Technology Studies (CWTS). http://www.leidenranking.com

Superintendencia de Industria y Comercio. (2017). Guía rápida de propiedad industrial. Issuu. https://issuu.com/quioscosic/docs/guia rapida pi

UE. (2017, marzo 15). Next-generation metrics: Responsible metrics and evaluation for open science. [Website]. Publications Office of the European Union. http://op.europa.eu/en/publication-detail/-/publication/b858d952-0a1911e7-8a35-01aa75ed71a1

Zhang, C.-T. (2009). The e-Index, Complementing the h-Index for Excess Citations. PLOS ONE, 4(5). https://doi.org/10.1371/journal.pone.0005429 


\section{Universidad Santo Tomás Boletín bibliométrico USTA \\ 2020}

